\title{
An implementation of the ray shooting method
}

\author{
Markus Hundertmark ${ }^{* \dagger}$ \\ Institut für Astrophysik, Georg-August-Universität Göttingen, Germany \\ E-mail: mhunder@astro.physik.uni-goettingen.de

\section{Frederic V. Hessman} \\ Institut für Astrophysik, Georg-August-Universität Göttingen, Germany \\ E-mail: hessmaneastro.physik.uni-goettingen.de
}

\section{Stefan Dreizler}

Institut für Astrophysik, Georg-August-Universität Göttingen, Germany E-mail: dreizlereastro.physik.uni-goettingen.de

\begin{abstract}
Ray shooting is a powerful but numerically costly means of solving the lens equation. Unlike pure numerical root finding techniques, ray shooting can automatically address the problem of finite size effects by defining the target area and, if necessary, weighting rays according to a limb-darkening model. This flexibility usually comes at the cost of calculating many - mostly unnecessary - rays. The method can be accelerated by isolating the areas of the lens plane required for each data point using targets distant from singularities. After an initial estimation of parameters, a pre-calculated grid of magnification patterns can then be searched for a global minimum of chi-square.
\end{abstract}

The Manchester Microlensing Conference: The 12th International Conference and ANGLES Microlensing Workshop

January 21-25 2008

Manchester, UK

\footnotetext{
* Speaker.

$\dagger$ I would like to thank the organizers of the Manchester Microlensing Conference for their great work and for the financial support from the ANGLES network.
} 


\section{Introduction}

The extraction of the physical properties of a microlensing event requires a model describing the accurate shape of the light curve. The total magnification can be obtained analytically if the Jacobian determinant of the lens equation is available at all image positions. In those cases where the image positions cannot be determined, numerical methods are required. One of them is the ray shooting method, relying on a grid of rays that can be deflected according to the lens equation. The magnification is proportional to the number of rays reaching a defined target corresponding to the source star.

The ray shooting technique was introduced by Kayser et al. (1986) for the simulation of Quasar microlensing, indicating that this method is especially suitable for the simulation of multiple lenses. Wambsganss (1997) showed that the ray shooting technique is also capable of simulating planetary light curves in galactic microlensing.

\section{Grid search on magnification maps}

As a first approach, a grid of 400 binary magnification maps, each covering an area of $25 \theta_{\mathrm{E}}^{2}$, was calculated with 2500 rays per pixel. Because of the axisymmetric magnification maps, about $30 \%$ of the rays can be neglected and each ray is randomly set in non-overlapping boxes.

For a grid of fixed mass ratio $q$ and lens separation $d\left[\theta_{\mathrm{E}}\right]$, the remaining parameters Einstein time $t_{\mathrm{E}}[\mathrm{d}]$, impact parameter $u_{\min }\left[\theta_{\mathrm{E}}\right]$, orientation angle $\beta\left[^{\circ}\right]$, and time of maximum magnification $t_{\max }[\mathrm{HJD}-245000]$ have been determined using the simplex method and simulated annealing as implemented in the $\mathrm{GSL}^{1}$.

The simplex method was carried out using a grid of initial values for $\beta \in\left[10^{\circ}, 170^{\circ}\right]$ and for $u_{\min } \in[-1: 1]\left[\theta_{\mathrm{E}}\right]$. Additionally, simulated annealing with 1000 tries per step, 500 iterations per fixed temperature, a step size of 3.0, a Boltzmann constant of 20, an initial temperature of 0.6 and $2 \%$ decrease of temperature has been tried. The success and speed of this method depends also sensitively on the configuration of the random walk. Finally, a single simplex is used to improve the final parameter estimation. The size of the target pixel was kept fixed at $0.0033 \theta_{\mathrm{E}}$, which roughly matches the finite size of the event OGLE-2003-BLG-170 as shown in Fig. 11.

Parameter uncertainties are estimated using the bootstrap method introduced by Efron (1979) for 500 resampled light curves. For computational reasons only the final simplex is searched repeatedly and provides estimations of uncertainties. The resulting scattered parameter estimations are not normally distributed and the uncertainties reported in Tab. 1 are given as 0.84 and 0.16 quantile displacements from the mode.

Because the resampled lightcurves are created by drawing values with replacement from the original dataset, features like a poorly sampled caustic crossing could be eliminated. The histograms that are shown in Fig. 2 indicate that a certain fraction of the final single simplex settles in different local minima caused by the $\chi^{2}$ structure and the resampling of the dataset.

\footnotetext{
${ }^{1}$ GNU Scientific Library
} 


\begin{tabular}{cccccccc}
\hline \hline Remarks & $u_{\min }$ & $t_{\mathrm{E}}$ & $t_{\max }$ & $\beta$ & $q$ & $d$ & $f$ \\
\hline Published $^{a}$ & -0.35 & 15.6 & 2794.1 & 133.66 & 0.789 & 1.213 & 0.75 \\
Simplex grid & -0.39 & 21.7 & 2793.6 & 122.4 & 0.85 & 1.3 & 0.6 \\
Annealing & -0.30 & 15.1 & 2793.8 & 134.1 & 0.55 & 1.2 & 0.74 \\
\hline Simplex & $-0.34_{-0.04}^{+0.03}$ & $15.7_{-0.5}^{+4.0}$ & $2793.3_{-0.1}^{+2.7}$ & $123.7_{-3.3}^{+5.9}$ & $0.8_{-0.2}^{+0.1}$ & $1.25_{-0.05}^{+0.15}$ & $0.83_{-0.23}^{+0.03}$ \\
\hline
\end{tabular}

${ }^{a}$ Jaroszynski et al. (2004)

Table 1: Test fits for OGLE-2003-BLG-170 using a pre-calculated grid
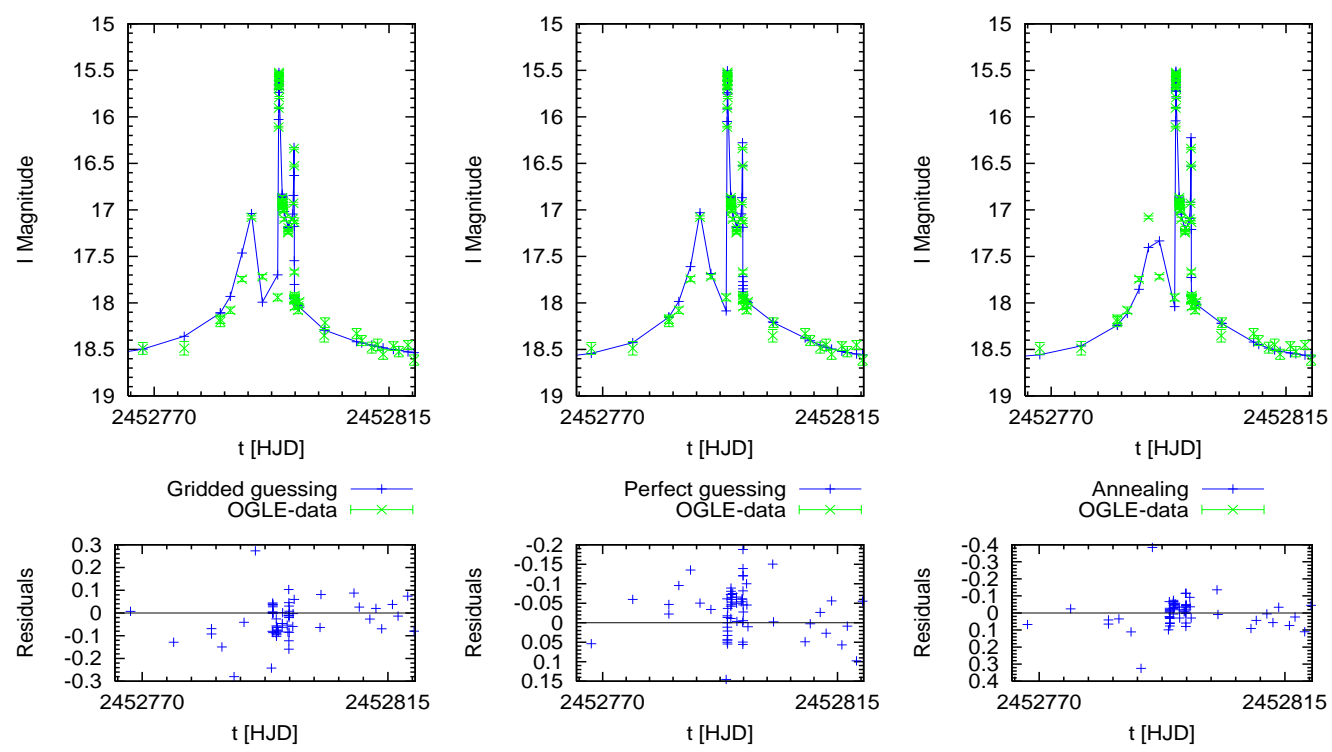

Figure 1: Fits for the binary microlensing event OGLE-2003-BLG-170 with different minimization methods along with the corresponding residuals are shown for grid of initial parameters for a simplex fit (left); for a single simplex fit (middle) and a simulated annealing fit (right).
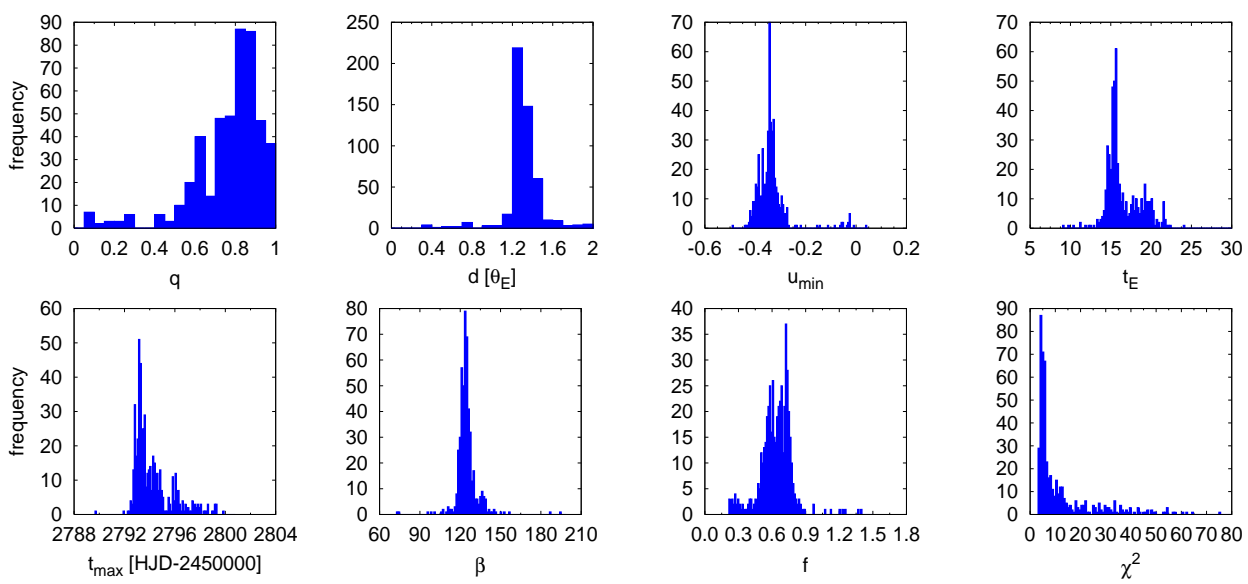

Figure 2: Histograms for the fitted parameters of the bootstrap resampled OGLE-2003-BLG-170 light curve are shown along with the distribution of the $\chi^{2}$ of the linear fit with a mode of 4.28. 


\section{Pointwise Ray shooting}

The ray shooting method can be accelerated by confining the grid of rays in the lens plane which has to be deflected. Fig. B shows the deflected grid for a point lens. The solutions of the lens equation can be calculated analytically for the corners of a box in which the source is located. The box size is chosen as in Rattenbury et al.(2002), assuring that the target is completely covered by rays. The definition of the corners fails near the lens position, which has to be compensated by an additional grid.

Defining the box vertices for binary lenses requires the solutions of the lens equation. This can be done by using the solutions of the 5th order polynomial as introduced by Witt \& Mao (1994). Alternatively the absolute deviation function for a defined source position, which is also be used for adaptive contouring (Dominik 2007), can be used to reduce the necessary grid. In Fig. 3 the minimum of a stack of deviation maps is plotted for source positions using the estimated parameters of OGLE-2003-BLG-170.
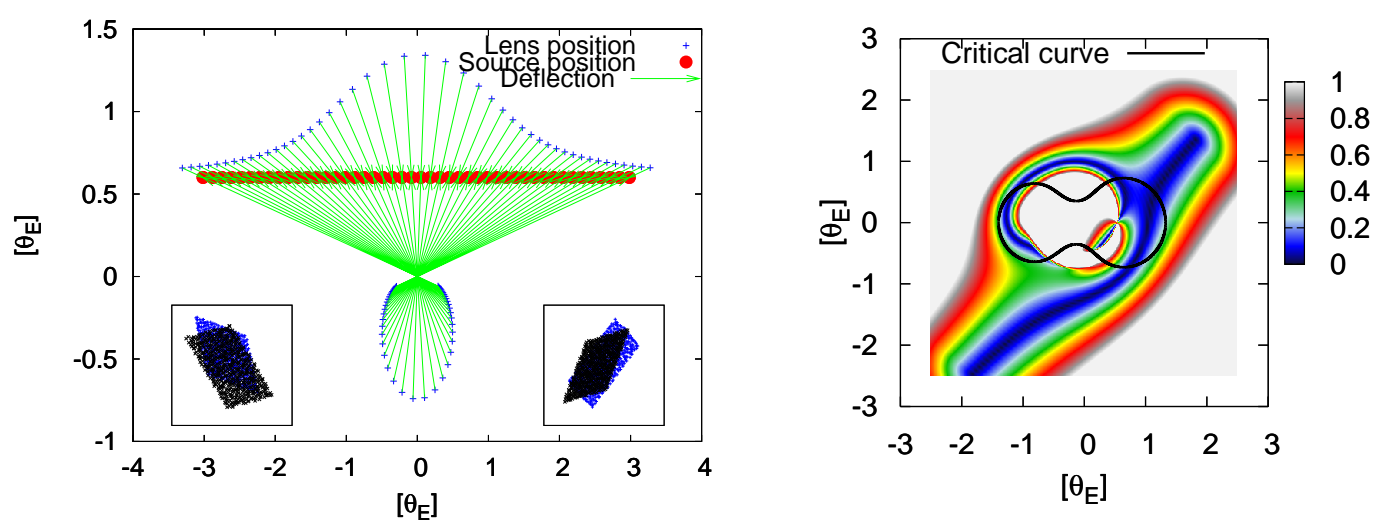

Figure 3: Pointwise Rayshooting for a point lens (left) and the minimal deviation function (Schramm \& Kayser 1987) of the source track of OGLE-2003-BLG-170 (right)

\section{Results of MONET test observations}

The feasibility of follow-up observations has been tested during the commissioning of the 1.2 $\mathrm{m}$ MONET/North telescope ( $30^{\circ}$ North, $104^{\circ}$ West). For this purpose the objects OGLE-2007BLG-006 and OGLE-2007-BLG-050 were selected from the Planet Lens Optimization page (cf. Snodgrass 2008) and were observed through 2 airmasses with an average seeing of $2.2 \operatorname{arcsec}$ for OGLE-2007-BLG-006 and 2.6 arcsec for OGLE-2007-BLG-050. The photometry of the MONET follow-up observation is carried out using the Difference Image Analysis Package ${ }^{2}$.

The OGLE-2007-BLG-006 light curve was fitted using the pre-calculated grid assuming a constant finite source size. The event OGLE-2007-BLG-050 were treated with the uniform finite size model introduced by Witt \& Mao (1994; for a detailed analysis of this event see Batista 2008). The results of our fits are shown in Tab. 2 and have been used to convert our data in Fig. 4 to the I-band magnitude reported by OGLE. The increased scatter of the last observations is caused by lower fluxes and observations after astronomical twilight.

\footnotetext{
${ }^{2}$ based on Wozniak (2000)
} 
OGLE-2007-BLG-006

\begin{tabular}{|c|c|c|c|c|c|c|}
\hline$u_{\min }$ & $t_{\mathrm{E}}$ & $t_{\max }$ & $\beta$ & $q$ & $d$ & $f$ \\
\hline $0.021_{-0.009}^{+0.001}$ & $33.9_{-0.5}^{+1.2}$ & $4172.6_{-0.8}^{+0.1}$ & $188.5_{-2.8}^{+0.3}$ & $0.275_{-0.075}^{+0.025}$ & $1.05_{-0.05}^{+0.05}$ & 1.0 \\
\hline \multicolumn{7}{|c|}{ OGLE-2007-BLG-050 } \\
\hline$u_{\min }$ & $t_{\mathrm{E}}$ & $t_{\max }$ & $r_{\mathrm{S}}$ & $f$ & & \\
\hline $0.0021_{-0.0002}^{+0.0002}$ & $65.9_{-1.9}^{+1.8}$ & $4221.974_{-0.003}^{+0.002}$ & $0.0044_{-0.0002}^{+0.0002}$ & $0.77_{-0.03}^{+0.02}$ & & \\
\hline
\end{tabular}

Table 2: Estimated parameters for OGLE OGLE-2007-BLG-005 and OGLE-2007-BLG-006
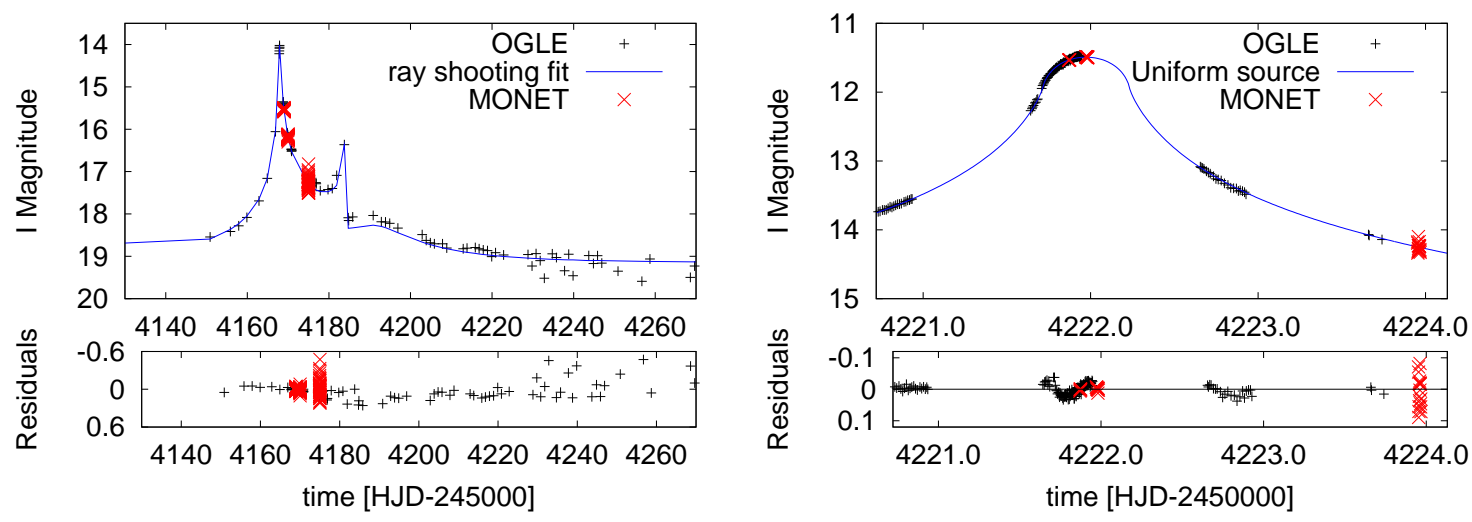

Figure 4: Light curves of OGLE-2007-BLG-006 (left) and OGLE-2007-BLG-050 (right)

\section{References}

[1] Batista, V., Detection efficiencies in high magnification microlensing events, in proceedings of The Manchester Microlensing Conference, PoS (GMC8) 059

[2] Dominik, M. 2007, MNRAS, 377, 1679 [astro-ph/0703305]

[3] Efron, B., 1979, Annals of Statistics, Vol. 7, 1-26

[4] Jaroszynski, M., et al. 2004, Acta Astronomica, 54, 103 [astro-ph/ 0408243 ]

[5] Kayser, R., Refsdal, S., \& Stabell, R. 1986, A\&A, 166, 36

[6] Rattenbury, N. J., Bond, I. A., Skuljan, J., \& Yock, P. C. M. 2002, MNRAS, 335, 159 [astro-ph/0204478]

[7] Schramm, T., \& Kayser, R. 1987, A\&A, 174, 361

[8] Snodgrass, C., The WEB-plop observation prioritisation system, in proceedings of The Manchester Microlensing Conference, POS (GMC8) 056

[9] Wambsganss, J. 1997, MNRAS, 284, 172 [astro-ph/9611134]

[10] Witt, H. J., \& Mao, S. 1994, ApJ, 430, 505

[11] Witt, H. J., \& Mao, S. 1995, ApJ, 447, L105

[12] Wozniak, P. R. 2000, Acta Astronomica, 50, 421 [astro-ph/0 012143 ] 